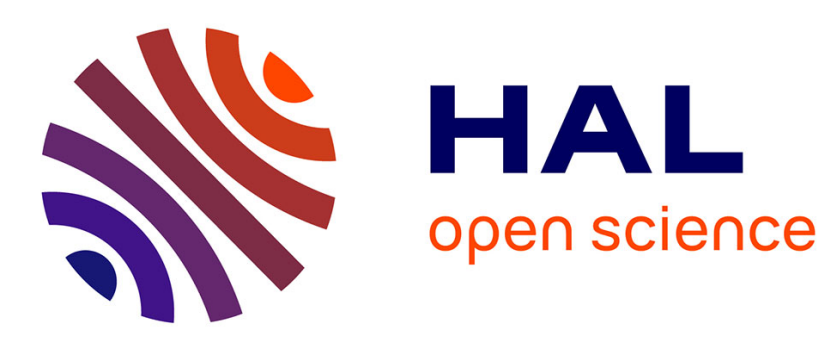

\title{
Design of a Micromachined CMOS Compass
}

Norbert Dumas, Laurent Latorre, Pascal Nouet

\section{To cite this version:}

Norbert Dumas, Laurent Latorre, Pascal Nouet. Design of a Micromachined CMOS Compass. TRANSDUCERS'05, Jun 2005, Séoul (Corée), pp.405-408. lirmm-00106457

\section{HAL Id: lirmm-00106457 https://hal-lirmm.ccsd.cnrs.fr/lirmm-00106457}

Submitted on 16 Oct 2006

HAL is a multi-disciplinary open access archive for the deposit and dissemination of scientific research documents, whether they are published or not. The documents may come from teaching and research institutions in France or abroad, or from public or private research centers.
L'archive ouverte pluridisciplinaire HAL, est destinée au dépôt et à la diffusion de documents scientifiques de niveau recherche, publiés ou non, émanant des établissements d'enseignement et de recherche français ou étrangers, des laboratoires publics ou privés. 


\title{
DESIGN OF A MICROMACHINED CMOS COMPASS
}

\author{
N. Dumas, L. Latorre and P. Nouet \\ LIRMM, Univ. Montpellier II / CNRS, Montpellier, France
}

\begin{abstract}
This paper presents the design of a low-cost monolithic CMOS micro-compass. The system is based on a cantilever actuated by the Lorenz force. Conditioning circuitry has been designed carefully to reject noise, to eliminate parasitic phenomenon such as thermal effects, and to be robust to process scatterings without the need of an external trimming. Mixed-mode simulations have been enabled using a behavioral description of the sensor, and validations of the complete system have been performed. The expected performances are a resolution inferior to $1^{\circ}$ for a total surface of $10.6 \mathrm{~mm}^{2}$ and a power consumption of $56 \mathrm{~mW}$ (without the digital processing bloc).
\end{abstract}

Keywords: MEMS design, compass, monolithic.

\section{INTRODUCTION}

Low-cost electronic compasses are used for vehicle navigation or antenna positioning. They can also be integrated in a cell phone or a watch to point the north direction on a display. A solution to build such an electronic compass consists in sensing the earth magnetic field along two directions in a horizontal plane.

Several products sense micro-Tesla magnetic field and then are suitable to make a compass system: AMR, GMR or fluxgate [1], [2]. A fully integrated 2-axis compass module, using AMR sensors, is even commercialized by Honeywell [3]. These devices are based on the use of a ferromagnetic layer and cannot be fabricated with a standard low-cost process. Hall Effect magnetic sensors provide a standard CMOS solution but are not enough sensitive for micro-compass applications [4].

The fabrication process of the proposed MEMS compass is based on the Front Side Bulk Micromachining of a standard CMOS die. It allows releasing reliable micromechanical frames at low-cost due to batch fabrication [5]. Monolithic integration of the electronics enables promising performances and additional functionalities such as analog to digital conversion or self-test. The sensing principle of the proposed device is a piezoresistive cantilever that is actuated with the Lorenz force. In a previous study [6], it has been reported that under specific operating conditions (i.e. Lorenz force actuation at resonant frequency), such a structure exhibits intrinsic performances (resolution, linearity) that make it practical to measure magnetic field below $1 \mu \mathrm{T}$, thus enabling compass applications.

In this context, a specific electronic architecture has been designed to actuate a cantilever at resonance and measure the output signal of the sensing part without degrading its resolution. Special cares have been given to make the architecture robust to process scattering (i.e. variation of the resonant frequency) without the need of external trimming. It is worth noting that the system design is well adapted for others MEMS sensors working at resonance, as for example resonant pressure sensors [7], micro-balance and humidity sensors.

The paper is organized as follows. In the first part, the sensing principle of the magnetic field sensor is described as well as its imperfections such as thermal parasitic effects. In the second part, the conditioning circuit is described for both the operating mode and the calibration/test mode. In the last part, the proposed design is validated by mixed-mode simulations.

\section{SENSOR BACKGROUND}

The sensing part of the compass is based on the piezoresistive U-Shaped cantilever presented in Fig. 1. The beam is composed of the back end layers of the CMOS process that are released by a wet etching post-process. The operating principle of the sensor relies on the interaction between the magnetic field $B$ to be measured and a known current If flowing through a planar coil embedded in the frame. The resulting Lorenz force actuates the structure. The stress induced in the structure near the anchor points is measured by means of embedded piezoresistive gauges in polysilicon. These gauges are placed in a Wheatstone bridge with two reference resistors on the substrate in order to transform the resistance variation into a voltage variation.

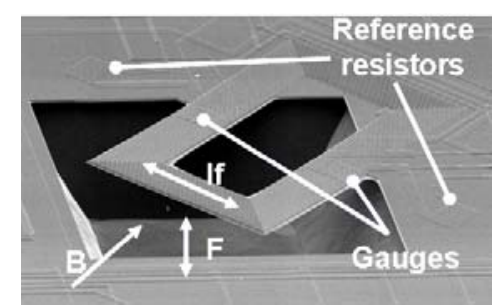

Fig. 1 SEM photograph of the magnetic field sensor

Experimental characterization of the device has shown that the mechanical structure reacts to the Lorenz force as a second-order low-pass system with a first resonant mode (fres) around $24 \mathrm{kHz}$ and a large quality factor of about 100 . To obtain the best sensor sensitivity $(1.2 \mathrm{~V} / \mathrm{T})$, the structure is actuated at resonance. Therefore, the operating mode of the sensor involves an AC bias current If applied across the aluminum coil.

For a constant low magnetic field (about $20 \mu \mathrm{T}$ ), the peak to peak amplitude of the Wheatstone bridge output 
(Vwheat) has been reported as a function of the frequency of the input sine wave current applied to the coil (Fig. 2). The expected resonance is found at $24 \mathrm{kHz}$ but other signals thermally induced are observed outside this resonant frequency. It is due to the power dissipated in structure that induces temperature variations. There are two effects: first, the polysilicon gauges are sensitive to the temperature. The cut-off frequency of this heating signal is about $20 \mathrm{~Hz}$; second a thermal actuation is induced because of the differences between all the thermal expansions coefficients of the structural materials. This actuation is only visible when it is amplified by the mechanical resonance of the cantilever. In fig.1.b this thermally induced resonance is observed for an input frequency of $12 \mathrm{kHz}$ (i.e. for a power varying at $24 \mathrm{kHz}$ ).

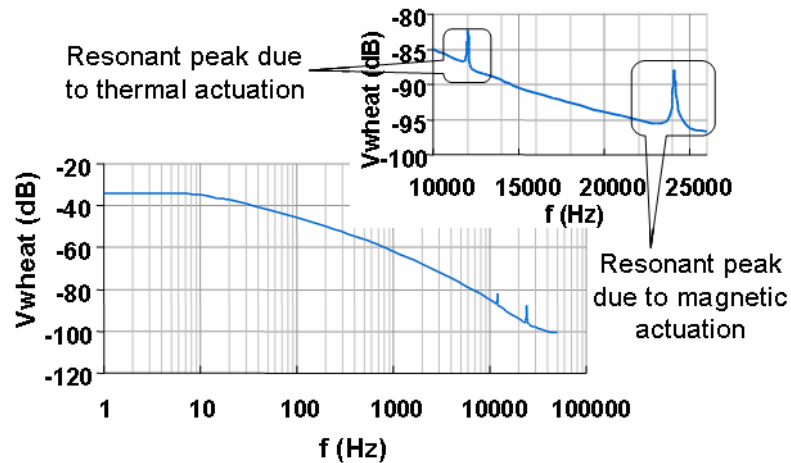

Fig. 2 Experimental harmonic response of a U-shaped cantilever to the applied current If

Thermal dissipation in the coil and the gauges also creates a DC offset at the output of the Wheatstone bridge. In addition, residual stress due the releasing process causes a total offset of about $50 \mathrm{mV}$. The Noise Equivalent Magnetic Induction (NEMI) is $5 \mu \mathrm{T}$ (considering a bandwidth of $500 \mathrm{kHz}$ ). It comes from the ratio between the voltage resolution at the output of the sensor and its sensitivity. This resolution is mainly limited by the thermal noise of the gauges and reference resistors.

\section{MICRO-COMPASS SYSTEM DESIGN}

\section{System architecture}

The system architecture of one sensing axis is presented on Fig. 3. Basically, it is composed of an excitation control block that drives the actuation coil, and a signal conditioning block that provides a measurement of the magnetic field.

The excitation control block must deliver a signal at the mechanical resonant frequency that is not precisely predictable. To adjust this actuation signal, open loop architecture has been preferred rather than an oscillator technique or a PLL [8]. These closed loop architectures cannot start oscillating at resonance when the magnetic field is low because the sensor signal is two low and exposed to noise. In open loop architecture, a calibration phase is then required to measure the resonant frequency.
It is activated by the $\mathrm{Cal} /$ test mode input (Fig. 3). A digital programmable frequency divider is used to deliver the excitation signal (If) in both modes (calibration and operating). During the calibration, the input frequency is scanned to find the resonant frequency. In operating mode, the input frequency is programmed to the resonant frequency.

Regarding the conditioning block on Fig. 3, lock-in amplifier architecture is used [9]. It is composed of an amplification chain (ampli \& offset filtering block), a switched capacitors band pass filter and a synchronous detection (Track and hold block). The band pass filter is originally auto-centered on the signal frequency by autoadjustment of the frequency of its clock (Switched capacitors clock signal).

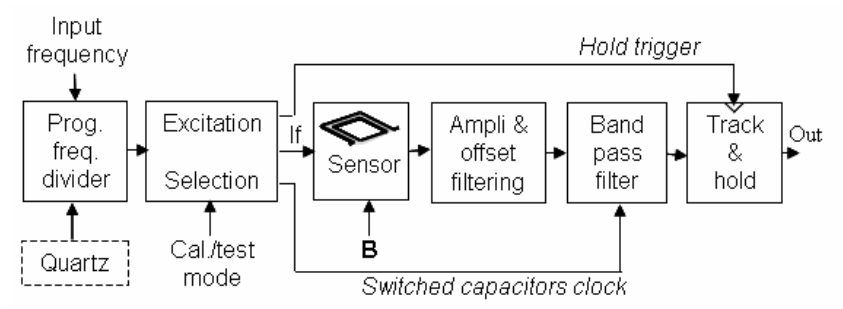

Fig. 3 System architecture of one sensing axis

The driving current (If) during operating mode is presented on Fig. 4. Using such a waveform, the power dissipated in the frame is constant and thermal effects only cause a DC offset at the output of the Wheatstone bridge. The $90^{\circ}$ phase shift between If and the output of the band pass filter is due to the mechanical resonance and the filter does not add phase shift. The peak amplitude is simply sampled by the track and hold circuit and analog to digital conversion is done during the hold phase.

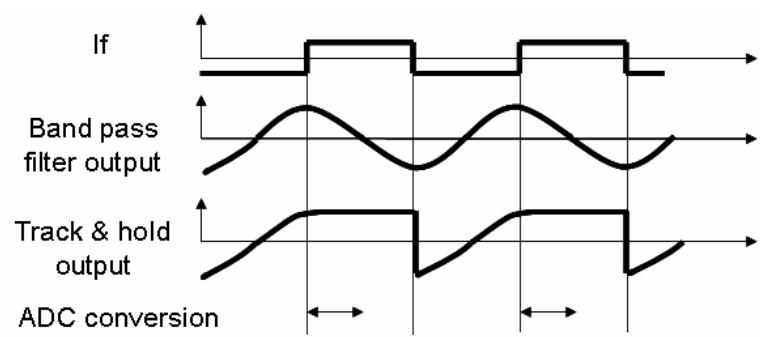

Fig. 4 Signals in operating mode

In calibration mode, the current applied to the coil of the cantilever is a 3-levels signal that can be considered as a very simplified sine wave. Temperature variations are generated by this way and a peak due to thermal actuation can be measured at half the resonant frequency.

Calibration is then fully electrical. There is no need to control the external magnetic field. The calibration mode has been presented for detecting faults in the device in [10]. Indeed, the presence of the resonant peak and the value of the resonant peak are necessary information to detect if the sensor is faulty. 


\section{Electronic design solutions}

In this section, the main blocks of the system architecture are detailed and design choices are commented in function of the sensor requirements.

The programmable frequency divider (Fig. 5) is based on the phase accumulator of a direct digital synthesizer generator [11]. The output is an $n$ bits word. The frequency and transition positions of these bits are useful to generate the signals If, Hold trigger and Switched capacitors clock (Fig. 3). Only a few gates are required in the excitation selection bloc. The frequency of each bit is given by:

$$
\text { freq. }\left(F d_{-} \text {out }[i]\right)=\text { Input freq. } \times \frac{\text { freq. }(\text { Quartz })}{2^{i}}
$$

The minimal frequency step is given for $i=n$. A frequency step equal to $40 \mathrm{~Hz}$ has been chosen for the thermal signal in calibration mode. So, it is guarantied that the actuation signal can fall in the bandwidth of mechanical resonance (about $200 \mathrm{~Hz}$ ). The frequency step of If is then $20 \mathrm{~Hz}$. For a quartz frequency equal to $20 \mathrm{Mhz}$, the number of bits $n$ must be 20. Each bit of Fd_out exhibits a large jitter. Fortunately, it has no incidence on the sensor performance because it is filtered by the mechanical response of the cantilever concerning If, and the response of the filter concerning Switched capacitors clock.

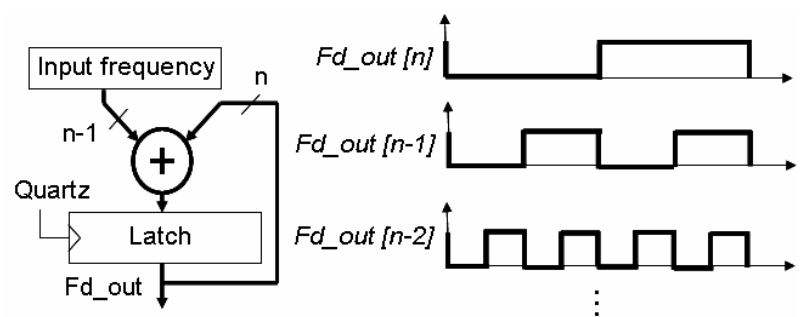

Fig. 5 Schematic of the digital frequency divider

The output of the sensor, i.e. the Wheatstone bridge, is first pre-amplified with a gain of 10. A low-noise amplifier (Fig. 6.a), based on differential pair, has been designed to preserve the resolution of the sensor $(33.2$ $\left.\mathrm{aV}^{2} / \sqrt{ } \mathrm{Hz}\right)$.

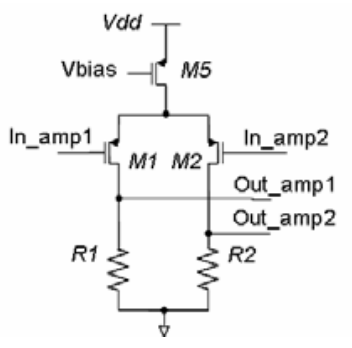

a

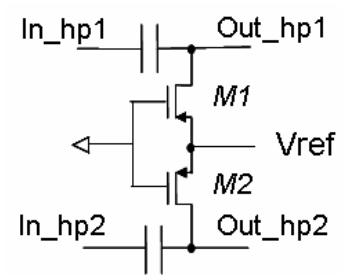

b
Fig. 6 Schematic of the low noise preamplifier (a) and the high pass filter (b)

Transistors $M 1$ and $M 2$ are sized large $(400 / 4 \mu \mathrm{m})$ in order to generate a total noise density of only 33.8 $\mathrm{aV}^{2} / \sqrt{\mathrm{Hz}}$ around $24 \mathrm{kHz}$ (mainly due to white noise). The gain is high enough to allow offset filtering with a $1 \mathrm{kHz}$ high pass filter (Fig. 6.b) without degrading the resolu- tion. In order to avoid the use of large passive components, both transistors $M 1$ and $M 2$ operate in triode region and have a resistance equal to $5 \mathrm{M} \Omega$. Offset filtering is necessary to prevent the following instrument amplifiers from saturation. The overall gain is 20,000 in order to adjust the signal dynamic with ADC requirements.

The band filter is used to eliminate noise and other undesired signal outside the bandwidth. It is made with switched capacitors techniques. It permits the adjustment of the center frequency (fc) by the clock signal. The frequency of the clock (Fclock) is 64 times the frequency of the signal to measure and is generated by bit number $i=$ 13 of the output of the frequency divider. As the center frequency is designed to be exactly 64 times less than the clock frequency (thanks to precise capacitors ratio), the filter is auto-centered. Main filter specifications are summed up in Table 1.

Table 1 Main filter specifications

\begin{tabular}{|c|l|c|}
\hline$\#$ & \multicolumn{1}{|c|}{ Specification description } & Value \\
\hline 1 & Bandwidth & $4 \mathrm{kHz}$ \\
2 & Attenuation at fc/2 & $30 \mathrm{~dB}$ \\
3 & Attenuation at fc $\times 2$ & $30 \mathrm{~dB}$ \\
4 & fc & Fclock $/ 64$ \\
5 & Maximum allowed loss of sensitivity, due to & $10 \%$ \\
& maladjustment of fc & $0^{\circ} \pm 10^{\circ}$ \\
\hline
\end{tabular}

Bandwidth specification is a compromise between resolution and precision of the capacitors ratio. Attenuation specifications are required to reject the crosstalk caused by the actuation signal. In operating mode, the undesired signal is found at $f c \times 2$. It is due to thermal phenomenon that is not fully rejected by the symmetry of If. In calibration mode, the undesired signal is found at $f c / 2$ and is due to capacitive coupling between the coil and the electronics. From this specification a two-section Butterworth structure has been retained. Because of the uncertainties of the capacitors ratio, tolerance margin on specification \#4 must be fixed. It is expressed in terms of specifications on the output magnitude (\#5) and on the output phase shift (\#6). Uncertainties about the phase shift reduce the sensitivity of the overall signal conditioning chain because the demodulation is done by synchronous detection.

\section{VALIDATIONS}

In order to validate the architecture proposed in previous section, a model of the sensor has been built. It takes into account the behavior of the sensor under magnetic and thermal stimuli but also represents non-idealities of the sensor.

High-level simulations have been run to validate the scanning of the input frequency in calibration mode (Fig. 7). As expected, the signal due to thermal actuation is the only measured signal and a resonant peak is found at half the resonant frequency. It is verified that the results are weakly influenced by magnetic fields lower than $1 \mathrm{mT}$. 


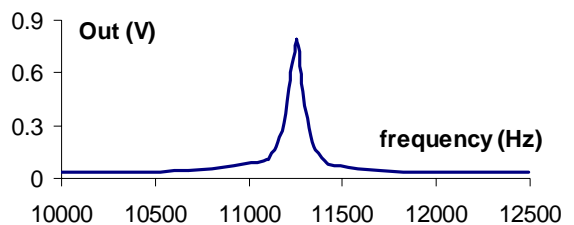

Fig. 7 Simulation of the frequency scanning in calibration mode

Each bloc of the compass architecture has been designed with the foundry design kit (AMS CMOS CYE $0.8 \mu \mathrm{m})$. The switched capacitors band pass filter has finally a bandwidth of $6 \mathrm{kHz}$, instead of $4 \mathrm{kHz}$, due to approximations of the capacitors. The NEMI of the sensor at the band pass filter output is calculated with a noise analysis and found to be $0.88 \mu \mathrm{T}$. Noise contribution of the gauges is $43 \%$. So, it is verified that the noise generated by the electronic components is not the main limit to the performance of the sensor.

Monte carlo analysis, taking into account process and mismatch, are necessary to verify the specifications \#5 and \#6 of Table 1. Simulations have been run with an input signal with a frequency 64 times lower than the frequency of the clock. The standard deviations found at the filter output are $0.15 \%$ for the magnitude, and $426 \mathrm{~m}^{\circ}$ for the phase. The specifications are then respected.

Other performances of the sensor with its signal conditioning chain are: a response time of $10 \mathrm{~ms}$ and a power consumption of $56 \mathrm{~mW}$ (with an ADC). The layout of the compass is presented on Fig. 8. The total surface is $10.6 \mathrm{~mm}^{2}$ (with the pads). Two U-shaped cantilevers are disposed orthogonally. Except for the preamplifiers, the electronic architecture of Fig. 3 is multiplexed for the two sensing axis.

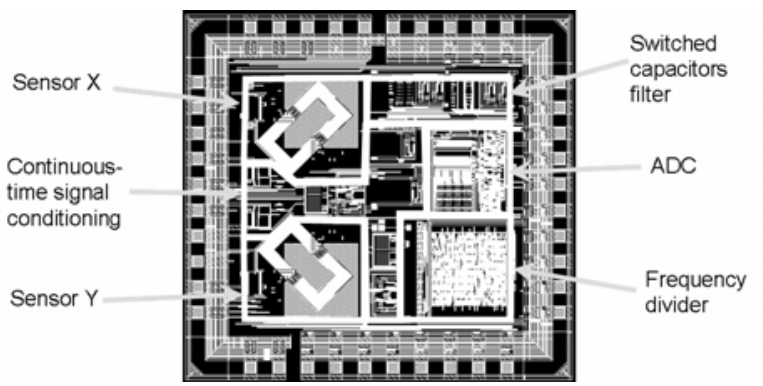

Fig. 8 Layout of the compass

For a complete compass system, digital electronics (specific or programmable) would be necessary to:

- Control the calibration phase used to find the resonant frequency.

- Compute the output angle with arctan function.

- Correct the difference of sensitivity between the two axis and reject offset due to magnetization of the environment. After a calibration phase consisting in a 360 turns of the die, coefficients of correction are calculated and used to compute the output angle.

- Filter the ADC output to enhance the resolution, if required. Without additional filtering, the resolution of the compass is about $3^{\circ}$. With a $50 \mathrm{~Hz}$ low pass filter the resolution is $0.1^{\circ}$ and the response time is $200 \mathrm{~ms}$ (fast enough for a lot of applications).

\section{CONCLUSION}

A monolithic compass has been presented. Its theoretical resolution is suitable (below $1^{\circ}$ ) for a lot of applications, including navigation system. The system architecture has been described. Two original solutions have been used to make the design robust to process scattering of the mechanical part. First the calibration adjusts the actuation signal to the resonant frequency thanks to thermal effects. Second, the filter, used for noise rejection, is auto-centered on the signal to measure. By this way its bandwidth can be narrow to enhance the performance of the compass. Mixed mode simulations validate the complete system and a circuit is currently under fabrication.

\section{REFERENCES}

[1] R. Tangelder, G. Diemel and H. Kerkhoff "Smart Sensor System Application: An Integrated Compass" Proceedings of the European Design and Test Conference, pp. 195-199, Paris, France, March 1997.

[2] H. Hauser, G. Stangl, W. Fallmann, R. Chabicovsky, K. Riedling, "Magnetoresistive Sensors" Proceedings of Workshop "Preparation, Properties, and Applications of Thin Ferromagnetic Films", pp. 15-27, Vienna, Austria, June 2000.

[3] M. J. Caruso, T. Bratland, C. H. Smith, R. Schneider, "Anisotropic Magnetoresistive Sensors Theory and Applications”, n`3, Volume 16, March 1999.

[4] H. Blanchard, F. De Montmollin, J. Hubin and R. S. Popovic, "Highly sensitive Hall sensor in CMOS technology", Sensors and Actuators A: Physical, pp. 144 - 148, Volume 82, Issues 1-3, 15 May 2000.

[5] M. Dardalhon, V. Beroulle, L. Latorre, P. Nouet, G. Perez, J.M. Nicot, C. Oudea, "Reliability analysis of CMOS MEMS structures obtained by Front Side Bulk Micromachining”, in Microelectronics reliability 42, pp 1777-1782, 2002.

[6] V. Beroulle, Y. Bertrand, L. Latorre, P. Nouet, "Monolithic piezoresistive CMOS magnetic field sensors", Sensors and actuators A 103 (2003) 23-32.

[7] O. Akar, T. Akin, T. Harpster, K. Najafi “A Wireless Batch Sealed Absolute Capacitive Pressure Sensor", Proceedings of The 14th European Conference on Solid-State Transducers (EUROSENSORS XIV), Copenhagen, Denmark, August 27-30, 2000.

[8] M. Aikele, K.Bauer, W. Ficker, F. Neuhauer, U. Prechtel, J.Sehalk, H. Seidel, "Resonant accelerometer with self-test", in Sensors and Actuator A 92, pp. 161-167, 2001.

[9] G. Ferri, P. De Laurentiis, A. D'Amico, C. Di Natale “A low voltage integrated CMOS analog lock in amplifier prototype for LAPS application", Sensors and Actuators A92, 263-272, 2001.

[10] N. Dumas, F. Azaïs, L. Latorre, P. Nouet, "On-Chip ElectroThermal Stimulus Generation for a MEMS-based Magnetic Field Sensor", accepted for publication in proceedings of VLSI Test Symposium (VTS'05).

[11] Antti Heiskanen, Antti Mäntyniemi, Timo Rahkonen, " $A 30 \mathrm{MHz}$ DDS Clock Generator with 8-bit, 130 ps Delay Generator and -50 $d B c$ Spurious Level", Proceedings of 27th European Solid-State Circuits Conference (ESSCIRC), Villach, Austria, 18-20 September 2001 . 